CERN-TH/96-87

FT/UCM/01/96

gr-qc/9604002

\title{
Normal Modes for Metric Fluctuations in a Class of Higher-Dimensional Backgrounds
}

\author{
M. Gasperini话 \\ Theory Division, CERN, CH-1211 Geneva 23, Switzerland \\ and \\ M. Giovanninif] \\ Departamento de Fisica Teorica, Universidad Complutense, 28040 Madrid, Spain
}

\begin{abstract}
We discuss a gauge invariant approach to the theory of cosmological perturbations in a higher-dimensonal background. We find the normal modes which diagonalize the perturbed action, for a scalar field minimally coupled to gravity, in a higher-dimensional manifold $\mathcal{M}$ of the Bianchi-type I, under the assumption that the translations along an isotropic spatial subsection of $\mathcal{M}$ are isometries of the full, perturbed background. We show that, in the absence of scalar field potential, the canonical variables for scalar and tensor metric perturbations satisfy exactly the same evolution equation, and we discuss the possible dependence of the spectrum on the number of internal dimensions.
\end{abstract}

\section{To appear in Class. Quantum Grav.}

CERN-TH/96-87

March 1996

\footnotetext{
${ }^{1}$ Permanent address: Dipartimento di Fisica Teorica, Un. di Torino, Via P. Giuria 1, 10125 Turin, Italy.

${ }^{2}$ Permanent address: DAMTP, Silver Street, Cambridge, CB3 9EW, UK.

${ }^{3}$ E-mail: M.Giovannini@damtp.cam.ac.uk
} 


\section{Introduction}

It is well known that the evolution in time of a classical cosmological background can amplify a given distribution of (initially small) inhomogeneous fluctuations of the metric and of the matter fields [il]. If the background is spatially homogeneous and isotropic, the fluctuations can be unambiguously classified as scalar, vector and tensor perturbations, according to their transformation properties under spatial coordinate transformations on a constant-time hypersurface (see for instance [2]). The components of such perturbations are not invariant under local infinitesimal coordinate transformations (also called "gauge" transformations); however, it is always possible to define variables that are "gauge-invariant" [3], at least to first order in the perturbation amplitude (see [⿴囗⿰丿㇄口] for a full covariant approach, gauge-invariant to all orders).

In a cosmological context, the initial conditions for the evolution in time of the perturbations are naturally provided by the quantum fluctuations of the metric and of the matter fields in their ground state [5]. The correct normalization of the perturbations to an initial vacuum fluctuation spectrum, however, requires the computation of the canonical variables that diagonalize the action (expanded to second order in the perturbation amplitude), and that represent the normal modes of oscillation of the matter-gravity coupled system [2] (see also 6, 7]). Such variables satisfy canonical commutation relations (or, classically, canonical Poisson brackets), and determine the absolute magnitude of the two-point correlation function for the perturbations, in terms of the vacuum fluctuation amplitude. The definition of these canonical variables is thus a necessary, preliminary step in order to study the time evolution of a primordial vacuum perturbation spectrum.

For a homogeneous and isotropic background, the canonical variable describing decoupled normal oscillations is known in the case of a perfect fluid source [8], and in the case of a scalar field source [9]. Attempts have been made to extend the canonical treatment to the case of two coupled scalar fields [10], but always in the context of an isotropic metric background. The main purpose of this paper is to relax the assumption of isotropy of the background manifold in the computation of the canonical normal modes of the Einstein-Hilbert action, minimally coupled to a scalar field source. We are motivated to perform this investigation by the development of models of the early Universe based on unified theories (such as the 
superstring theory [11]), where the background manifold is usually given by the product of a $(d+1)$-dimensional "external" space-time and of an $n$-dimensional "internal" manifold, whose shape and radial size are possibly time-dependent.

In anisotropic backgrounds, a gauge-invariant description of metric perturbations is complicated by the coupling among modes with different rotational transformation properties, and relative to different spatial subsections (for instance, a coupling between "external" scalars and "internal" tensors becomes allowed, in principle). A possible approach to this problem [12], valid when the background $\mathcal{M}$ can be factorized as the product of two (or more) isotropic sub-manifolds $\mathcal{V}_{i}$ (i.e. $\mathcal{M}=\mathcal{V}_{1} \otimes \mathcal{V}_{2} \otimes \ldots$ ), is to define gauge-invariant variables in each of the sub-manifolds $\mathcal{V}_{i}$. In that context the fluctuations may be classified as scalars, vectors and tensors with respect to coordinate transformations in the "physical" external sub-manifold. A different approach [13], which can be applied to any Bianchi-type I metric background, is to define gauge-invariant variables with respect to infinitesimal coordinate transformations defined on the whole manifold. In that case one obtains a very complicated system of perturbed equations which, up to now, has been solved only under the simplifying assumptions that the propagation is restricted to an effective $(2+1)$-dimensional space-time [14].

The approach of the present paper is more similar, in spirit, to that of [12]. We shall assume that the translations along the internal dimensions are isometries of the full, perturbed metric background. Under this assumption, we shall compute the canonical variables for the normal oscillations of a background which is the product of two conformally flat manifolds. Our result shows that in this case a very simple action, invariant under global $S U(2) \otimes U(1)$ transformations, can simultaneously account for the scalar and tensor linear fluctuations of the metric tensor.

The assumption that perturbations depend only on the external coordinates is justified in the context of a typical Kaluza-Klein background describing a phase of dynamical dimensional reduction 15, 16, 17], in which the accelerated inflationary expansion of the external dimensions, with scale factor $a$, is sustained by the accelerated contraction of the internal ones, with scale factor $b$. In that case, in fact, the curvature scales of the internal and external manifolds are both growing in time: if we accept, as it seems natural [16, 17], that at 
the end of the process (at a conformal time $\eta_{1}$ ) internal and external curvature scale are of the same (nearly Planckian) order, i.e. $\left(a_{1} \eta_{1}\right)^{-1} \sim\left(b_{1} \eta_{1}\right)^{-1}$, then $a \ll b$ for all $\eta<\eta_{1}$. Since $(a / b)^{2}$ controls the ratio of internal to external gradients in the equations determining the time evolution of the perturbations, it follows that the dependence on the internal coordinates tends to be suppressed for large enough perturbation scales, namely for all modes $k$ crossing the horizon well before $\eta_{1}$, when the contribution of internal gradients can be safely neglected. This is what happens, in particular, in the scalar field-dominated background that we analyze in this paper.

The paper is organized as follows. In Section 2 we present the coupled evolution equations for the scalar perturbation variables, in a generalized longitudinal gauge. The equations are directly obtained by perturbing the equations of motion for the metric and scalar field background. In Section 3 we expand the perturbed action up to second order, and we introduce the gauge-invariant variables that reduce the action to the diagonal, canonical form. As an application of our results, we discuss the possible dependence of the tensor perturbation spectrum on the number of internal dimensions. A brief summary, and our concluding remarks, are finally presented in Section 4.

\section{Background equations and scalar perturbations}

We start with the $D$-dimensional action for a scalar field, minimally coupled to gravity:

$$
S=S_{g}+S_{m}=-\frac{1}{6 l_{D}^{2}} \int d^{D} x \sqrt{-g} R+\int d^{D} x \sqrt{-g}\left[\frac{1}{2} g^{\alpha \beta} \partial_{\alpha} \varphi \partial_{\beta} \varphi-V(\varphi)\right]
$$

where $l_{D}=\sqrt{8 \pi G_{D} / 3}$ has dimensions of length to the power $(D-2) / 2$. We shall consider a homogeneous, Bianchi-type I metric background, whose spatial part is the product of two conformally flat manifolds:

$$
\begin{array}{r}
g_{\mu \nu}=\operatorname{diag}\left(a^{2}(\eta),-a^{2}(\eta) \delta_{i j},-b^{2}(\eta) \delta_{m n}\right), \quad \varphi=\varphi(\eta), \\
\mu, \nu=0, \ldots, D-1=d+n, \quad i, j=1, \ldots, d, \quad m, n=d+1, \ldots, d+n,
\end{array}
$$

and $\eta$ is the conformal time coordinate (the main results of this paper, however, can be easily generalized to the case of $d+n$ different scale factors). For such background, the equations 
of motion obtained by varying the action with respect to $g_{\mu \nu}$ and $\varphi$,

$$
R_{\mu}^{\nu}-\frac{1}{2} \delta_{\mu}^{\nu} R=3 l_{D}^{2}\left[\partial_{\mu} \varphi \partial^{\nu} \varphi-\frac{1}{2} \delta_{\mu}^{\nu} g^{\alpha \beta} \partial_{\alpha} \varphi \partial_{\beta} \varphi+\delta_{\mu}^{\nu} V(\varphi)\right], \quad g^{\alpha \beta} \nabla_{\alpha} \nabla_{\beta} \varphi+\frac{\partial V}{\partial \varphi}=0
$$

reduce simply to

$$
\begin{gathered}
d(d-1) \mathcal{H}^{2}+n(n-1) \mathcal{F}^{2}+2 n d \mathcal{H} \mathcal{F}=6 l_{D}^{2}\left(\frac{\varphi^{\prime 2}}{2}+a^{2} V\right) \\
2(d-1) \mathcal{H}^{\prime}+(d-1)(d-2) \mathcal{H}^{2}+2 n \mathcal{F}^{\prime}+n(n+1) \mathcal{F}^{2}+2 n(d-2) \mathcal{H} \mathcal{F}=6 l_{D}^{2}\left(a^{2} V-\frac{\varphi^{\prime 2}}{2}\right) \\
2(n-1) \mathcal{F}^{\prime}+2 d \mathcal{H}^{\prime}+d(d-1) \mathcal{H}^{2}+n(n-1) \mathcal{F}^{2}+2(d-1)(n-1) \mathcal{H} \mathcal{F}=6 l_{D}^{2}\left(a^{2} V-\frac{\varphi^{\prime 2}}{2}\right) \\
\varphi^{\prime \prime}+[(d-1) \mathcal{H}+n \mathcal{F}] \varphi^{\prime}+\frac{\partial V}{\partial \varphi}=0,
\end{gathered}
$$

where $\mathcal{H}=(\ln a)^{\prime}, \mathcal{F}=(\ln b)^{\prime}$ and a prime denotes differentiation with respect to $\eta$. These equations are not all independent, and the scalar field equation, for instance, can be obtained from the other Einstein equations. For $\varphi=0$ these equations describe a particular KaluzaKlein vacuum [15], and are solved by a higher-dimensional generalization of the well-known Kasner metric background.

By summing and subtracting the above equations one obtains

$$
\begin{aligned}
6 l_{D}^{2} a^{2} V= & {[(d-1) \mathcal{H}+n \mathcal{F}]^{\prime}+[(d-1) \mathcal{H}+n \mathcal{F}]^{2} } \\
3 l_{D}^{2} \varphi^{\prime 2}= & -[(d-1) \mathcal{H}+n \mathcal{F}]^{\prime}+(d-1) \mathcal{H}^{2}-n \mathcal{F}^{2}+2 n \mathcal{H} \mathcal{F}, \\
& \mathcal{F}^{\prime}-\mathcal{H}^{\prime}=-(\mathcal{F}-\mathcal{H})[(d-1) \mathcal{H}+n \mathcal{F}]
\end{aligned}
$$

We shall discuss in this paper the case in which the contribution of the scalar potential to the background equations is negligible, which is for instance a reasonable approximation for the dilaton-driven phase of string cosmology [18, 19. In this case, by setting $V(\varphi)=0$, the combination of eqs. (2.5) and (2.6) provides the relation

$$
(\ln \mathcal{H})^{\prime}=(\ln \mathcal{F})^{\prime}=-[n \mathcal{F}+(d-1) \mathcal{H}]
$$

which will prove very useful for the computation of the perturbed action, in the next section.

We now expand metric and scalar field perturbations around a solution of the background equations (2.3), assuming that all dynamical variables depend only on the "external" coordinates $x^{i}, i=1, \ldots, d$. In this case modes with different rotational transformation properties 
are decoupled, and the scalar component of the background perturbations can be written in general as

$$
g_{\mu \nu} \rightarrow g_{\mu \nu}(\eta)+\delta g_{\mu \nu}^{(S)}\left(x^{i}, \eta\right), \quad \varphi \rightarrow \varphi(\eta)+\chi\left(x^{i}, \eta\right)
$$

where

$$
\delta g_{\mu \nu}^{(S)}=\left(\begin{array}{ccc}
2 a^{2} \phi & -a^{2} B_{i} & 0 \\
-a^{2} B_{i} & 2 a^{2} \psi \delta_{i j}-2 a^{2} E_{i j} & 0 \\
0 & 0 & 2 b^{2} \xi \delta_{a b}
\end{array}\right)
$$

(notations: $\left.B_{i}=\partial_{i} B, E_{i j}=\partial_{i} \partial_{j} E\right)$, and all variables $(\phi, \psi, \xi, E, B)$ depend only on $\eta$ and $x^{i}$. Under an infinitesimal coordinate transformation, preserving the scalar nature of the fluctuation [2],

$$
x^{i} \rightarrow \tilde{x}^{i}=x^{i}+\partial^{i} \epsilon\left(\eta, x^{i}\right), \quad \eta \rightarrow \tilde{\eta}=\eta+\epsilon^{0}\left(\eta, x^{i}\right)
$$

the components of scalar perturbations transform as

$$
\begin{aligned}
& \phi \rightarrow \tilde{\phi}=\phi-\mathcal{H} \epsilon^{0}-\epsilon^{0^{\prime}} \\
& \psi \rightarrow \tilde{\psi}=\psi+\mathcal{H} \epsilon^{0} \\
& \xi \rightarrow \tilde{\xi}=\xi+\mathcal{F} \epsilon^{0} \\
& E \rightarrow \tilde{E}=E-\epsilon \\
& B \rightarrow \tilde{B}=B+\epsilon^{0}-\epsilon^{\prime} \\
& \chi \rightarrow \tilde{\chi}=\chi-\varphi^{\prime} \epsilon^{0}
\end{aligned}
$$

A possible choice of "gauge-invariant" (linearly independent) variables is then:

$$
\begin{array}{rlrl}
\Phi & =\phi+\frac{1}{a}\left[\left(B-E^{\prime}\right) a\right]^{\prime}, & \Psi & =\psi-\mathcal{H}\left(B-E^{\prime}\right), \\
\Xi & =\xi-\mathcal{F}\left(B-E^{\prime}\right), & X=\chi+\varphi^{\prime}\left(B-E^{\prime}\right) .
\end{array}
$$

We choose in this paper $\tilde{B}=0$ and $\tilde{E}=0$, which defines a generalized "longitudinal" (or conformally Newtonian) gauge [2], and which leaves the coordinate system completely fixed. By perturbing, in this gauge, the background equations (2.3) (with $V=0$ ) we obtain the first-order equations for the classical evolution of the scalar inhomogeneities. The $(i, j)$ component, $i \neq j$, of the perturbed Einstein equations gives a relation between the three perturbation variables,

$$
\phi=(d-2) \psi+n \xi
$$


This allows eliminating $\phi$ everywhere in the perturbation equations. The $(0,0)$ component gives

$$
(d-1) \nabla^{2} \psi+n \nabla^{2} \xi-\psi^{\prime}[d(d-1) \mathcal{H}+n d \mathcal{F}]-n[d \mathcal{H}+(n-1) \mathcal{F}] \xi^{\prime}=3 l_{D}^{2} \varphi^{\prime} \chi^{\prime}
$$

The $(i, i)$ components give

$$
\begin{array}{r}
(d-1) \psi^{\prime \prime}+\psi^{\prime}[(d-1)(2 d-3) \mathcal{H}+n(2 d-3) \mathcal{F}]+n \xi^{\prime \prime}+ \\
+\xi^{\prime}\left[2 n(d-1) \mathcal{H}+\left(2 n^{2}+n\right) \mathcal{F}-n \mathcal{H}\right]=3 l_{D}^{2} \varphi^{\prime} \chi^{\prime}
\end{array}
$$

The $(m, m)$ components give

$$
\begin{aligned}
d \psi^{\prime \prime}+(n-1) \xi^{\prime \prime} & +\nabla^{2} \xi-\nabla^{2} \psi+\psi^{\prime}[2 d(d-1) \mathcal{H}+2(d-1)(n-1) \mathcal{F}]+ \\
& +\xi^{\prime}[(d(2 n-1)-(n-1)) \mathcal{H}+2 n(n-1) \mathcal{F}]=3 l_{D}^{2} \varphi^{\prime} \chi^{\prime}
\end{aligned}
$$

The scalar field equation gives

$$
\chi^{\prime \prime}+[(d-1) \mathcal{H}+n \mathcal{F}] \chi^{\prime}-\nabla^{2} \chi=2 \varphi^{\prime}\left[(d-1) \psi^{\prime}+n \xi^{\prime}\right]
$$

Finally, the $(0, i)$ components of the Einstein equations give the constraint:

$$
(d-1) \psi^{\prime}+(d-2) \psi[(d-1) \mathcal{H}+n \mathcal{F}]+n \xi^{\prime}+n \xi[(n+1) \mathcal{F}+(d-2) \mathcal{H}]=3 l_{D}^{2} \varphi^{\prime} \chi
$$

All these perturbation equations have been obtained by using the explicit form (2.4) of the background equations.

It is now convenient to define the variable

$$
\lambda=\psi+\frac{n}{d-1} \xi
$$

which satisfies the equation

$$
\square \lambda+3[(d-1) \mathcal{H}+n \mathcal{F}] \lambda^{\prime}=0
$$

$\left(\square=(\partial / \partial \eta)^{2}-\nabla^{2}\right)$ obtained by subtracting eq. (2.15) from eq. (2.14). The combination of eqs. 2.16) and (2.14) gives

$$
\begin{aligned}
& d\left\{\square \psi+\psi^{\prime}\left[3(d-1) \mathcal{H}+\frac{\mathcal{F}}{d}(2(d-1)(n-1)+n d)\right]\right\}= \\
= & -(n-1)\left\{\square \xi+\xi^{\prime}\left[\frac{\mathcal{H}}{n-1}(3 d n-d-n+1)+3 n \mathcal{F}\right]\right\} .
\end{aligned}
$$


By inserting in this equation the expression for $\square \psi$ obtained from (2.20) we obtain:

$$
\square \xi+[3 n \mathcal{F}+(d-1) \mathcal{H}] \xi^{\prime}+2(d-1) \mathcal{F} \psi^{\prime}=0 \quad .
$$

Finally, by eliminating $\square \xi$ by means of the above equation, we obtain from eq. (2.20)

$$
\square \psi+[3(d-1) \mathcal{H}+n \mathcal{F}] \psi^{\prime}+2 n \mathcal{H} \xi^{\prime}=0 \quad .
$$

The system of equations (2.22) and (2.23) describes the coupled evolution of the "external" and "internal" scalar perturbation variables, $\psi$ and $\xi$. By contrast, each polarization mode of tensor perturbations is decoupled from the others. A pure (transverse, traceless) tensor fluctuation $h$ of the $d \times d$ external part of the metric background satisfies the free scalar field equation, minimally coupled to the geometry [20, 21],

$$
h^{\prime \prime}+[(d-1) \mathcal{H}+n \mathcal{F}] h^{\prime}-\nabla^{2} h=0,
$$

and is automatically invariant under infinitesimal coordinate transformations preserving the tensor nature of the fluctuations. The similarities between the time evolution of scalar and tensor perturbations will become more explicit, however, when comparing the canonical variables, which diagonalize the action (2.1), expanded up to second order in the amplitude of the metric fluctuations. This will be done in the next section.

\section{Normal modes for canonical oscillations}

In this section we shall expand the action (2.1) up to second order in the amplitude of scalar fluctuations, and we shall look for the "normal coordinates", i.e. for the canonical (gaugeinvariant) variables which diagonalize the perturbed action. To this aim, it is convenient to express the gravitational part of the action, $S_{g r}$, in terms of the Adler-Deser-Misner (ADM) formalism [22], in such a way that the second derivatives of the metric tensor appear only as a total derivative [23]. By setting

$$
d s^{2}=\left(N^{2}-N_{\alpha} N^{\alpha}\right) d \eta^{2}-2 N_{\alpha} d x^{\alpha} d \eta-\gamma_{\alpha \beta} d x^{\alpha} d x^{\beta},
$$

where Greek indices (only in this section) run from 1 to $d+n$, we obtain

$$
S_{g r}=\frac{1}{6 l_{D}^{2}} \int d^{D} x\left[N \sqrt{\gamma}\left(K_{\beta}^{\alpha} K_{\alpha}^{\beta}-K^{2}\right)+\frac{1}{2}\left(\sqrt{\gamma} \gamma^{\alpha \beta} N\right)_{, \alpha}(\ln \gamma)_{, \beta}+N_{, \alpha}\left(\sqrt{\gamma} \gamma^{\alpha \beta}\right)_{, \beta}\right]-
$$




$$
-\frac{1}{6 l_{D}^{2}} \int d^{D} x\left[\frac{1}{2} N \bar{\Gamma}_{\alpha \beta}^{\gamma} \sqrt{\gamma} \gamma_{, \gamma}^{\alpha \beta}-\mathcal{D}_{(1)}\right]
$$

where

$$
\begin{gathered}
\mathcal{D}_{(1)}=-2(K \sqrt{\gamma})^{\prime}+2\left(\sqrt{\gamma} K N^{\alpha}-\sqrt{\gamma} \gamma^{\alpha \beta} N_{, \beta}\right)_{, \alpha}-\left[N \gamma^{\alpha \beta}(\sqrt{\gamma})_{, \beta}+\left(N \sqrt{\gamma} \gamma^{\alpha \beta}\right)_{, \beta}\right]_{, \alpha}, \\
K_{\alpha \beta}=\frac{1}{2} N^{-1}\left(\nabla_{\alpha} N_{\beta}+\nabla_{\beta} N_{\alpha}-\gamma_{\alpha \beta}^{\prime}\right), \quad K=K_{\alpha}^{\alpha} .
\end{gathered}
$$

Here a comma denotes partial differentiation; $N_{\alpha}$ are the shift vectors; $N$ is the lapse function; $\gamma^{\alpha \beta}$ is the spatial $(d+n)$-dimensional part of the metric tensor; $\bar{\Gamma}$ is the corresponding Christoffel connection; $\gamma=\operatorname{det} \gamma_{\alpha \beta} ; K_{\alpha}^{\beta}$ is the extrinsic curvature of the $(d+n)$-dimensional, $\eta=$ const hypersurface. Finally, indices are raised, and covariant derivatives are computed, with the spatial metric $\gamma_{\alpha \beta}$.

Comparing eq. (3.1) with the perturbed form of the metric tensor, eq. (2.9), we can easily express the ADM functions up to second order in the perturbation variables as:

$$
\begin{aligned}
& N=a\left(1+\phi-\frac{1}{2} \phi^{2}+\frac{1}{2} B_{i} B_{i}\right), \quad N_{i}=a^{2} B_{i}, \quad N_{m}=0, \\
& \gamma^{i j}=a^{2}(1-2 \psi) \delta_{i j}+2 a^{2} E_{i j}, \quad \gamma_{m n}=b^{2}(1-2 \xi) \delta_{m n}, \\
& \operatorname{det}\left(\gamma_{\alpha \beta}\right)=\operatorname{det}\left(\gamma_{i j} \otimes \gamma_{m n}\right)=a^{2 d} b^{2 n}\left[1-2 n \xi-2 d \psi+2 E_{i i}+2 n(n-1) \xi^{2}+\right. \\
& \left.+4 n d \psi \xi-4 n E_{i i} \xi+2 d(d-1) \psi^{2}-4(d-1) \psi E_{i i}+2 E_{i i} E_{j j}-2 E_{i j} E_{i j}\right] .
\end{aligned}
$$

By using these definitions in eq. (3.2) we obtain the gravity part of the action, expanded to second order in the scalar perturbation amplitude. With a similar technique it is also possible to write the matter part of the action, up to second order in the amplitude of the fluctuations of the scalar field $(\chi)$ and of metric. The result of this long algebraic procedure gives the full-second order action:

$$
\begin{aligned}
\delta_{(2)} S & =\delta_{(2)} S_{g r}+\delta_{(2)} S_{m}=\frac{1}{6 l_{D}^{2}} \int d^{D} x a^{d-1} b^{n}\left\{-d(d-1) \psi^{\prime 2}-\right. \\
& -n(n-1) \xi^{\prime 2}-2 d(d-1) \mathcal{H} \phi \psi^{\prime}+(d-1)\left[(d-2) \psi_{i}-2 \phi_{i}\right] \psi_{i}- \\
& -2 n(n-2)[(n-1) \mathcal{F}+d \mathcal{H}] \xi \xi^{\prime}-2 n[(n-1) \mathcal{F}+d \mathcal{H}] \phi \xi^{\prime}-2 n d \psi^{\prime} \xi^{\prime}- \\
& -2 n d[n \mathcal{F}+(d-1) \mathcal{H}] \xi \psi^{\prime}+2 n \xi_{i}\left(2 \psi_{i}+\frac{n-1}{2} \xi_{i}-\phi_{i}\right)+
\end{aligned}
$$




$$
\begin{aligned}
& +6 l_{D}^{2}\left[\left(\varphi^{\prime}\left(\phi^{\prime}+d \psi^{\prime}+n \xi^{\prime}\right) \chi+\frac{1}{2} \chi^{\prime 2}-\frac{1}{2} \chi_{i}^{2}\right]+4\left(B-E^{\prime}\right)_{i i}\left[-\frac{n}{2} \mathcal{F} \xi-\frac{n(d-1)}{2} \mathcal{H} \xi-\right.\right. \\
& \left.\left.-\quad \frac{n}{2} \xi^{\prime}-\frac{n}{2} \mathcal{F} \phi+\frac{n d}{2} \mathcal{H} \xi-\frac{d-1}{2} \mathcal{H} \phi-\frac{d-1}{2} \mathcal{H} \psi^{\prime}+\frac{3}{2} l_{D}^{2} \chi \varphi^{\prime}\right]\right\}+\frac{1}{6 l_{D}^{2}} \int d^{D} x \mathcal{D}_{(2)}(3.6)
\end{aligned}
$$

where the total derivative $\mathcal{D}_{(2)}$ is given explicitly by

$$
\begin{aligned}
\mathcal{D}_{(2)} & =\partial_{i}\left\{a ^ { d - 1 } b ^ { n } \left[-4(d-1) \mathcal{H}\left(E_{i j}\left(B-E^{\prime}\right)_{j}-E_{j j}\left(B-E^{\prime}\right)_{i}+\frac{1}{2} E_{j j} B_{i}\right)+\right.\right. \\
& +\left(B-E^{\prime}\right)_{i j}\left(B-E^{\prime}\right)_{j}-\left(B-E^{\prime}\right)_{j j}\left(B-E^{\prime}\right)_{i}+E_{i j l} E_{j l}-E_{j j l} E_{l i}- \\
& -2 n(n-1) \mathcal{F} \xi B_{i}-2 n \mathcal{F} \psi B_{i}-6 n \mathcal{H} \xi B_{i}-n(n-1) \mathcal{F}^{2}\left(E_{j j} E_{i}-E_{j} E_{j i}\right)- \\
& -2 n(d \mathcal{H}+(n-1) \mathcal{F}) \xi B_{i}+(d-2)(n \mathcal{F}-2(d-1) \mathcal{H}) \psi B_{i}-6 l_{D}^{2} \varphi^{\prime} \chi B_{i}+ \\
& +4 n \mathcal{F}\left(\left(B-E^{\prime}\right)_{i} E_{j j}-\left(B-E^{\prime}\right)_{j} E_{j i}\right)+6 n \mathcal{H} \mathcal{F}\left(E_{j i} E_{j}-E_{j j} E_{i}\right)+ \\
& \left.\left.+d(d-1) \mathcal{H}^{2}\left(E_{i j} E_{j}-E_{j j} E_{i}\right)\right]\right\}+ \\
& +\partial_{\eta}\left\{a ^ { d - 1 } b ^ { n } \left[2 n(d-2)(\mathcal{F}+(d-1) \mathcal{H}) \psi E_{i i}+2 n d(\mathcal{H}+\mathcal{F}) \xi E_{i i}+6 l_{D}^{2} \varphi^{\prime} \chi E_{i i}+\right.\right. \\
& +2((d-1) \mathcal{H}+n \mathcal{F}) E_{i i} E_{j j}-d((d-1) \mathcal{H}+n(d-2) \mathcal{F}) \psi^{2}- \\
& \left.\left.-6 l_{D}^{2} \varphi^{\prime}(\phi+d \psi+n \xi) \chi\right]\right\} .
\end{aligned}
$$

In order to obtain the previous expressions we have repeatedly used the background equations (2.4) and the relation (2.7). The functional derivative of the action (3.6) with respect to $\left(B-E^{\prime}\right)$ provides a constraint [2] which relates the different longitudinal fluctuations:

$$
3 l_{D}^{2} \chi \varphi^{\prime}=[(d-1) \mathcal{H}+n \mathcal{F}] \phi+(d-1) \psi^{\prime}+n \xi^{\prime}+n(\mathcal{F}-\mathcal{H}) \xi .
$$

By using eq. (2.13) this expression reduces exactly to the constraint (2.18), which we obtained in the previous section from the $(0, i)$ component of the perturbed Einstein equations. This is quite an important consistency check of our procedure.

By inserting eq. (3.8) in the second-order action (3.6), we can finally diagonalize this action by introducing the following two variables $v$ and $w$ :

$$
\begin{gathered}
v=a^{\frac{d-1}{2}} b^{\frac{n}{2}} \chi+z \lambda=a^{\frac{d-1}{2}} b^{\frac{n}{2}} X+z \Lambda, \\
w=\frac{z}{l_{D}}\left[\frac{n(n+d-1)}{6(d-1)}\right]^{1 / 2}\left(\frac{\mathcal{H}}{\varphi^{\prime}} \xi-\frac{\mathcal{F}}{\varphi^{\prime}} \psi\right)=\frac{z}{l_{D}}\left[\frac{n(n+d-1)}{6(d-1)}\right]^{1 / 2}\left(\frac{\mathcal{H}}{\varphi^{\prime}} \Xi-\frac{\mathcal{F}}{\varphi^{\prime}} \Psi\right),
\end{gathered}
$$

where

$$
z=\frac{a^{\frac{d-1}{2}} b^{\frac{n}{2}} \varphi^{\prime}}{\mathcal{H}+\frac{n}{d-1} \mathcal{F}}, \quad \Lambda=\Psi+\frac{n}{d-1} \Xi
$$


The gauge invariance of $w$ and $v$ is simply a consequence of the gauge invariance of the variables $\Psi, X, \Xi$, defined in eq. (2.12). In terms of $v$ and $w$ the action (3.6) can be written in the canonical form as the action for two non-interacting scalar fields, both coupled to the same time-dependent external potential $z^{\prime \prime} / z$, namely

$$
\delta_{(2)} S=\frac{1}{2} \int d^{D} x\left[v^{\prime 2}+\frac{z^{\prime \prime}}{z} v^{2}-v_{i}^{2}+w^{\prime 2}+\frac{z^{\prime \prime}}{z} w^{2}-w_{i}^{2}+2 \mathcal{D}_{(3)}\right]
$$

where

$$
\begin{aligned}
\mathcal{D}_{(3)} & =\frac{d}{d \eta}\left\{\frac{d-1}{6 l_{D}^{2}} \frac{a^{d-1} b^{n} \lambda_{i}^{2}}{\mathcal{H}+\frac{n}{d-1} \mathcal{F}}-\frac{v^{2}}{2}\left(\frac{d-1}{2}\right)\left(\mathcal{H}+\frac{n}{d-1} \mathcal{F}\right)-\frac{3}{d-1} \frac{l_{D}^{2} \varphi^{\prime 2} v^{2}}{a^{\frac{d-1}{2}} b^{\frac{n}{2}}}+\right. \\
& +\frac{3}{2} \frac{l_{D}^{2} z^{2} \varphi^{\prime}}{a^{\frac{d-1}{2}} b^{\frac{n}{2}}} \lambda v-\frac{n}{d-1}[(n-1) \mathcal{F}+d \mathcal{H}] z \xi v+\frac{n}{d-1}[(n-1) \mathcal{F}+d \mathcal{H}] z^{2} \lambda \xi+ \\
& +\frac{3}{2(d-1)} \frac{l_{D}^{2} \varphi^{\prime}}{a^{\frac{d-1}{2}} b^{\frac{n}{2}}} z^{3} \lambda^{2}+d z z^{\prime}(\lambda \psi)-d \psi v z^{\prime}-\frac{n d}{3} \frac{a^{d-1} b^{n}}{l_{D}^{2}}[(n-1) \mathcal{F}+d \mathcal{H}] \xi^{2}+ \\
& \left.+\frac{n^{2}}{6(d-1)} \frac{a^{d-1} b^{n}}{l_{D}^{2}} \frac{(\mathcal{H}-\mathcal{F})[(n-1) \mathcal{F}+d \mathcal{H}]}{(d-1) \mathcal{H}+n \mathcal{F}} \xi^{2}\right\}
\end{aligned}
$$

is another total derivative, which does not contribute to the equations of motion.

The two variables $v$ and $w$ generalize to the higher-dimensional, anisotropic case $d>3$, $n \neq 0$, the canonical variable [9] representing normal oscillations in $d=3, n=0$, introduced for a gauge-invariant description of scalar perturbations. In the absence of scalar field, the equations describing the fluctuations of our particular Kaluza-Klein vacuum [15] can be directly obtained by setting $\varphi=\chi=0$ in the corresponding equations of Section 2. In this case there is only one normal mode of oscillation, and the action reduces to the canonical form (3.12) with $v=0$ and

$$
w=\left[\frac{n(n+d-1)}{6 l_{D}^{2}(d-1)}\right]^{1 / 2} \frac{a^{\frac{d-1}{2}} b^{\frac{n}{2}}}{\mathcal{H}+\frac{n}{d-1} \mathcal{F}}(\mathcal{H} \xi-\mathcal{F} \psi)
$$

(the isotropic, $d$-dimensional case is recovered for $b=$ const, $\mathcal{F}=0$ ). If we have, on the contrary, $N>1$ scalar fields minimally coupled to gravity,

$$
S=-\frac{1}{6 l_{D}^{2}} \int d^{D} x \sqrt{-g} R+\frac{1}{2} \int d^{D} x \sqrt{-g}\left[\sum_{k=1}^{N} g^{\alpha \beta} \partial_{\alpha} \varphi_{k} \partial_{\beta} \varphi_{k}\right]
$$

the action (3.12) easily generalizes to the action describing the oscillations of $N+1$ normal modes, with canonical variables

$$
w, \quad v_{k}=a^{\frac{d-1}{2}} b^{\frac{n}{2}} \chi_{k}+z_{k} \lambda, \quad z_{k}=\frac{a^{\frac{d-1}{2}} b^{\frac{n}{2}} \varphi_{k}^{\prime}}{\mathcal{H}+\frac{n}{d-1} \mathcal{F}}, \quad k=1, \ldots, N
$$


where $\chi_{k}=\delta \varphi_{k}$, and $w$ is the same variable as in eq. (3.10). For $n=0$ and $d=3$, this result coincides with the one recently obtained in [24]. The results of this section cannot be directly applied to the case in which the scalar potential $V(\varphi)$ is non-vanishing, since in that case eq. (2.7) is no longer valid and the expressions for the total derivative terms become more complicated.

According to the action (3.12), the Fourier components of the canonical variables $v$ and $w$ satisfy the evolution equations

$$
v_{k}^{\prime \prime}+\left[k^{2}-\frac{z^{\prime \prime}}{z}\right] v_{k}=0, \quad w_{k}^{\prime \prime}+\left[k^{2}-\frac{z^{\prime \prime}}{z}\right] w_{k}=0 .
$$

These equations also directly follow from the definition of $v$ and $w$, and from the evolution equations of the scalar fluctuations derived in the previous section, namely eqs. (2.17), (2.22) and (2.23). In particular, for a power-like behaviour of the background, $z(\eta) \sim|\eta|^{\alpha}$, eqs. (3.17) are solved exactly by

$$
\begin{aligned}
& v_{k}=\frac{1}{\sqrt{k}}\left[A_{k} \sqrt{|k \eta|} H_{\nu}^{(2)}(|k \eta|)+B_{k} \sqrt{|k \eta|} H_{\nu}^{(1)}(|k \eta|)\right], \\
& w_{k}=\frac{1}{\sqrt{k}}\left[C_{k} \sqrt{|k \eta|} H_{\nu}^{(2)}(|k \eta|)+D_{k} \sqrt{|k \eta|} H_{\nu}^{(1)}(|k \eta|)\right],
\end{aligned}
$$

where $\nu=|\alpha-1 / 2|$, and $H_{\nu}^{(1)}, H_{\nu}^{(2)}$ are the first- and second-kind Hankel functions [25]. Once that $v$ and $w$ are known, the Fourier components of the metric perturbation variables, $\psi_{k}, \xi_{k}$, can be expressed in terms of the gauge-invariant variables as

$$
\begin{aligned}
k^{2} \psi_{k} & =\frac{n(n+d-1) \mathcal{H} \mathcal{F} \varphi^{\prime}}{[(d-1) \mathcal{H}+n \mathcal{F}]^{2}}\left[\frac{6 l_{D}^{2}(d-1)}{n(n+d-1)}\right]^{1 / 2}\left(\frac{w_{k}}{z}\right)^{\prime}- \\
& -\frac{3 l_{D}^{2} \varphi^{\prime} \mathcal{H}}{[(d-1) \mathcal{H}+n \mathcal{F}]}\left(\frac{v_{k}}{a^{\frac{d-1}{2}} b^{\frac{n}{2}}}\right)^{\prime}-\frac{n \varphi^{\prime}}{(d-1) \mathcal{H}+n \mathcal{F}}\left[\frac{6 l_{D}^{2}(d-1)}{n(n+d-1)}\right]^{1 / 2}\left(\frac{w_{k}}{z}\right), \\
\xi_{k} & =\frac{d-1}{n}\left(\lambda_{k}-\psi_{k}\right), \\
k^{2} \lambda_{k} & =\frac{n \mathcal{F}}{d-1} \frac{\left[(n+d-1) \varphi^{\prime}\right]}{[(d-1) \mathcal{H}+n \mathcal{F}]}\left[\frac{6 l_{D}^{2}(d-1)}{n(n+d-1)}\right]^{1 / 2}\left(\frac{w}{z}\right)^{\prime}-\frac{3 l_{D}^{2} \varphi^{\prime}}{(d-1)}\left(\frac{v}{a^{\frac{d-1}{2}} b^{\frac{n}{2}}}\right)^{\prime}
\end{aligned}
$$

(we have used the whole set of equations (2.14)-(2.17)). For $n=0, d=3, \xi_{k}=0$, we recover the standard relation [2]

$$
\psi_{k}=-\frac{3 l_{D}^{2}}{2} \frac{\varphi^{\prime}}{k^{2}}\left(\frac{v}{a}\right)^{\prime}
$$


It is now interesting to compare, at the level of canonical variables, the behaviour of scalar perturbations with that of tensor perturbations propagating in a $d=3$ external metric background. In terms of the two transverse and traceless tensor polarization modes, $h_{\oplus}$ and $h_{\otimes}$, the second-order action for tensor perturbations, up to a total derivative, can be written

$$
\delta_{(2)} S^{(T)}=\frac{1}{2} \int d^{4+n} x\left[u_{\oplus}^{\prime 2}+u_{\otimes}^{\prime 2}+\frac{y^{\prime \prime}}{y}\left(u_{\oplus}^{2}+u_{\otimes}^{2}\right)-\left(\partial_{i} u_{\oplus}\right)^{2}-\left(\partial_{i} u_{\otimes}\right)^{2}\right],
$$

where the canonical variables $u_{\oplus}$ and $u_{\otimes}$ are defined by [19, 20, 26]

$$
u_{\oplus}=\frac{y}{24 l_{D}} h_{\oplus}, \quad u_{\otimes}=\frac{y}{24 l_{D}} h_{\otimes}, \quad y=a b^{n / 2} .
$$

In the absence of scalar potential we have $y^{\prime \prime} / y=z^{\prime \prime} / z$. We can thus rewrite the sum of the two actions (3.12) and (3.21) in compact form, invariant under global $S U(2) \otimes U(1)$ transformations:

$$
\delta_{(2)} S^{(S)}+\delta_{(2)} S^{(T)}=\int d^{4+n} x\left[\eta^{\mu \nu}\left(\partial_{\mu} \mathcal{Q}\right)^{\dagger} \partial_{\nu} \mathcal{Q}-m^{2} \mathcal{Q}^{\dagger} \mathcal{Q}\right]
$$

where

$$
\mathcal{Q}=\left(\begin{array}{c}
q \\
Q
\end{array}\right), \quad q=\frac{v+i w}{\sqrt{2}}, \quad Q=\frac{u_{\oplus}+i u_{\otimes}}{\sqrt{2}}, \quad m^{2}=-\frac{z^{\prime \prime}}{z}=-\frac{y^{\prime \prime}}{y},
$$

and $\eta_{\mu \nu}$ is the flat Minkowski metric in $4+n$ dimensions. This action explicitly displays the similarities in the time evolution of the gauge-invariant, canonical variables for scalar and tensor fluctuations, in spite of the very different equations of motion for the metric perturbation variables, written in the longitudinal gauge. This similar behaviour was also stressed in [19], where it was argued that the gauge-invariant variables could be more appropriate than the standard longitudinal ones for a consistent expansion of scalar inhomogeneities outside the horizon.

As a physical application of our results, we shall discuss the possible dependence of the tensor perturbation spectrum on the number of internal dimensions, by considering a background with $d=3$ and $n \neq 0$ (a similar discussion can be easily repeated in the case of scalar perturbations). The power spectrum $P_{h}(k)$ is defined as usual [27] in terms of the Fourier transform of the two-point correlation function,

$$
\left\langle h\left(x_{i}\right) h\left(x_{i}^{\prime}\right)\right\rangle=\int \frac{d^{3} k}{(2 \pi k)^{3}} e^{i k_{i}\left(x_{i}-x_{i}^{\prime}\right)} P_{h}(k),
$$


where the brackets denote spatial average, or ensamble average over a distribution of stochastic variables. For each perturbation mode $h_{k}$ we thus have, from eq. (3.22),

$$
P_{h}(k) \simeq k^{3}\left|h_{k}\right|^{2} \simeq \frac{k^{3}}{M_{p}^{n}} \frac{\left|u_{k}\right|^{2}}{\left(y M_{p}\right)^{2}}
$$

modulo numerical factors of order unity $\left(M_{p}\right.$ is the Planck mass). According to the action (3.21), the canonical variable $u_{k}$ satisfies exactly the free evolution equation (3.17), with $z$ replaced by $y$. In a power-law background with $y \sim|\eta|^{\alpha}$ we thus choose as solution

$$
u_{k}=|\eta|^{1 / 2} H_{\nu}^{(2)}(|k \eta|), \quad \nu=|\alpha-1 / 2|,
$$

which satisfies the correct vacuum normalization [2] for $|k \eta| \gg 1$. In the opposite limit $|k \eta| \ll 1$ the perturbations are amplified by the background evolution, and eq. (3.27) gives $\left|u_{k}\right| \simeq|k \eta|^{\alpha} / \sqrt{k}=y /(\sqrt{k} y)_{h c}$, where " $h c$ " denotes the time of horizon crossing, $\eta=k^{-1}$ (we have assumed $\alpha<1 / 2$, corresponding to the case in which the comoving amplitude of perturbations stay frozen outside the horizon; for a discussion of the opposite case see for instance [28]). Since $y^{2}=a^{2} b^{n}$ in $d=3+n$, this gives the normalized spectrum as

$$
P_{h}(k) \simeq M_{p}^{-n}\left(y^{-2}\right)_{h c}\left(\frac{k}{M_{p}}\right)^{2} \simeq\left(\frac{k}{a M_{p}}\right)_{h c}^{2}\left(b M_{p}\right)_{h c}^{-n} \simeq\left(\frac{H}{M_{p}}\right)_{h c}^{2}\left(b M_{p}\right)_{h c}^{-n}
$$

$(H=d(\ln a) / d t$ is the usual Hubble parameter defined with respect to cosmic time $t)$.

When $n=0$ we thus recover the standard tensor perturbation spectrum, determined by the Hubble factor at horizon crossing; when $n \neq 0$ the spectrum seems instead to be affected by the dynamics of the internal dimensions. For the scalar field-dominated background of this paper, however, the modification is only apparent, because from the general solution of the background equations [18] we have $y^{2}=a^{2} b^{n} \sim|\eta|$, i.e. $\alpha=1 / 2$. The $n$-dependence disappears from $y$ so that, according to eq. (3.28), $P_{h}(k) \sim k^{2+2 \alpha} \sim k^{3}$ (modulo logarithmic corrections) like in a four-dimensional background [18, 19, quite independently of $n$.

The situation is obviously different when other sources are present, beside the scalar field. Consider, for instance, the addition of perfect fluid matter, with energy density $\rho$ and pressure $p_{1}=\gamma_{1} \rho, p_{2}=\gamma_{2} \rho$, in the external and internal submanifolds, respectively. Tensor perturbations are decoupled from the fluid sources, so that the canonical variables are the same, and the normalized spectrum is still given by eq. (3.28). From the general solution of 
the background equations with perfect fluids, given in [18, we get (in the Einstein frame):

$$
y^{2}=a^{2} b^{n} \sim|\eta|^{2 \alpha}, \quad 2 \alpha=\frac{2\left(1-\gamma_{1}\right)}{1-2 \gamma_{1}+3 \gamma_{1}^{2}+n \gamma_{2}^{2}} .
$$

The spectrum (3.28), $P_{h}(k) \sim k^{2+2 \alpha}$, is now $n$-dependent (unless we put $\gamma_{2}=0$ ), and the contribution of the internal dimensions goes in the direction of flattening the spectrum with respect to the pure $d=3$ case.

\section{Discussion and conclusion}

In this paper we discuss a Lagrangian approach to the perturbations of scalar field matter, minimally coupled to gravity in a homogeneous cosmological background. We explicitly consider a metric of the Kaluza-Klein type, corresponding to the direct product of two conformally flat (internal and external) manifolds. Under the assumption of frozen dependence on the internal coordinates (namely for perturbations only depending on the variables of one of the two spatial sub-manifolds), we find the canonical variables that diagonalize the original action, up to second order in the amplitude of the metric and scalar field fluctuations. The total action can then be written in a compact form in terms of a complex bivector, which provides a unified description of scalar and tensor normal excitations of the background manifold.

This suggests that, in a string cosmology context, the $O(d+n, d+n)$ covariance of the background equations [29] should characterize not only the evolution of tensor perturbations [30, but that of scalar perturbations (in the String frame) as well. In the Einstein frame, used in this paper, it is interesting to observe that the time evolution of the scalar and tensor canonical variables is determined by the background function

$$
\frac{z^{\prime \prime}}{z}=\frac{y^{\prime \prime}}{y}=-\left(\frac{d-1}{2}\right)^{2}\left(\mathcal{H}+\frac{n \mathcal{F}}{d-1}\right)^{2}=-\left(\frac{z^{\prime}}{z}\right)^{2},
$$

which is invariant under the transformation $a \rightarrow a^{-1}, b \rightarrow b^{-1}$, which implies $z \rightarrow-z^{-1}$. It is also invariant, separately, under the transformation $\varphi \rightarrow-\varphi$, which implies $z \rightarrow-z$. In the Einstein frame this second transformation corresponds indeed, in the absence of potential for the scalar field, to a scale factor duality transformation [31 for an isotropic, dilaton-driven 
background, evolving in time with a power-like behaviour [18]. If the background admits, in particular, an asymptotic regime in which the Laplacian terms become negligible in the evolution equations, then the invariance of $z^{\prime \prime} / z$ implies the invariance, in that regime, of $q^{\prime \prime} / q$ and $Q^{\prime \prime} / Q$ as a functions of $z$.

As already mentioned in the Introduction, and stressed in [2, 6, 7], the correct normalization of the metric perturbations to an initial vacuum fluctuation spectrum is only possible after introducing the gauge-invariant variables representing the normal modes, which diagonalize the action and satisfy canonical commutation relations. This paper should be regarded as a first step towards the definition of such variables in the case of higher-dimensional backgrounds. It would be important, however, to drop the assumption that fluctuations depend only on the external coordinates. The dependence on internal coordinates modifies in fact the perturbation equations: for the tensor case, by adopting for instance the approach of [12], one obtains

$$
\begin{array}{r}
h_{i}^{j^{\prime \prime}}+[(d-1) \mathcal{H}+n \mathcal{F}] h_{i}^{j^{\prime}}-\nabla_{x}^{2} h_{i}^{j}-\frac{a^{2}}{b^{2}} \nabla_{y}^{2} h_{i}^{j}=0, \\
h_{a}^{b^{\prime \prime}}+[(d-1) \mathcal{H}+n \mathcal{F}] h_{a}^{b^{\prime}}-\nabla_{x}^{2} h_{a}^{b}-\frac{a^{2}}{b^{2}} \nabla_{y}^{2} h_{a}^{b}=0,
\end{array}
$$

where $\nabla_{x}^{2}$ and $\nabla_{y}^{2}$ are, respectively, the external and internal Laplacian operator; $h_{i}^{j}$ and $h_{a}^{b}$ are the external and internal polarization modes. Unless the terms with the internal Laplacian, asymptotically, become sub-leading with respect to the others, the dependence on the internal coordinates can modify in a significant way the power spectrum computed under the assumption that such coordinates are frozen.

Finally, it would be interesting to repeat the analysis of this paper in the context of the Hamiltonian formalism, more appropriate for the discussion of the constraints that characterize scalar perturbations, and for the standard approach to the canonical quantization procedure of the fluctuations.

\section{Acknowledgements}

We are grateful to Gabriele Veneziano for many useful discussions. M. Giovannini wishes to thank the CERN Theory Division for hospitality during the completion of this research. 
This work is supported in part by the "Human Capital and Mobility Program" of the European Commission, under the contracts No. CHRX-CT94-0423 (M. Giovannini) and No. ERBCHRX-CT94-0488 (M. Gasperini). 


\section{References}

[1] Lifschitz E M 1946 Zh. Eksp. Teor. Fiz. 16 587; Lifschitz E M and Khalatnikov I 1963 Adv. Phys. 12 185; Grishchuk L P 1975 Sov. Phys. JEPT 40 409; Starobinski A A 1979 JEPT Lett. 30682

[2] Mukhanov V F, Feldman H A and Brandenberger R H 1992 Phys. Rep. 215203

[3] Bardeen J M 1980 Phys. Rev. D 22 1882; Sasaki M 1983 Prog. Theor. Phys. 70394

[4] Ellis G F R and Bruni M 1989 Phys. Rev. D 401804

[5] Sakharov A D 1966 Sov. Phys. JETP 2241

[6] Grishchuk L P 1992 in Proceeding of the Sixth Marcel Grossmann Meeting, Kyoto, 1991, ed. by H. Sato (World Scientific, Singapore)

[7] Deruelle N Gundlach G and Polarski D 1992 Class. Quantum Grav. 9137

[8] Lukash V N 1980 Zh. Eksp. Teor. Fiz. 79 1601; Chibisov G V and Mukhanov V F 1982 Mon. Not. R. Astron. Soc. 200535

[9] Sasaki M 1986 Prog. Theor. Phys. 76 1036; Mukhanov V F 1988 Zh. Eksp. Teor. Fiz. 94 1; Stewart E D and Lyth D H 1983 Phys. Lett. B 302171

[10] Deruelle N, Gundlach C and D. Langlois 1992 Phys. Rev. D 45 R3301; Deruelle N, Gundlach C and Langlois D 1993 Phys. Rev. D 465337

[11] Green M B, Schwartz J H and Witten E 1987 Superstring Theory (Cambridge University Press, Cambridge)

[12] Abbott R B, Bednarz B and Ellis S D 1986 Phys. Rev. D 332147

[13] Tomita K and Den M 1986 Phys. Rev. D 343570

[14] Den M 1987 Prog. Theor. Phys. 77653

[15] Appelquist T, Chodos A and Freund P G O 1987 Modern Kaluza-Klein Theories (Addison-Wesley, Redwood City) 
[16] Abbot R B Barr S M and Ellis S D 1984 Phys. Rev. D 30 720; Kolb E W, Lindley D and Seckel D 1984 Phys. Rev. D 301205.

[17] Gasperini M and Veneziano G 1993 Astropart. Phys. 1317.

[18] Gasperini M and Veneziano G 1994 Phys. Rev. D 502519

[19] Brustein R, Gasperini M, Giovannini M, Mukhanov V F and Veneziano G 1995 Phys. Rev. D 516744

[20] Gasperini M and Giovannini M 1992 Class. Quantum Grav. 9 L137; Gasperini M and Giovannini M 1993 Phys. Rev. D 471519

[21] Demianski M, Polnarev A G and Naselski P 1993 Phys. Rev. D 475275

[22] Arnowitt R, Deser S and Misner C W 1962 in Gravitation: an introduction to current research, ed. by L. Witten (Wiley, New York)

[23] Fock V 1964 The theory of space, time and gravitation (Pergamon Press, Oxford)

[24] Anderegg S and Mukhanov V F 1994 Phys. Lett. B 33130

[25] Abramowitz M and Stegun I A 1972 Handbook of mathematical functions (Dover, New York)

[26] Giovannini M 1995 Quantum theory of perturbations in string cosmology, PhD Thesis (University of Turin) (unpublished)

[27] Liddle A R and Lyth D H 1993 Phys. Rep 2311.

[28] Gasperini M 1995 in Third Paris Cosmology Colloquium ed De Vega H J and Sanchez N (World Scientific, Singapore) p 253.

[29] Meissner K A and Veneziano G 1991 Mod. Phys. Lett. A 6 3397; Gasperini M and Veneziano G 1992 Phys. Lett. B 277256

[30] Brustein R, Gasperini M, Giovannini M and Veneziano G 1995 Phys. Lett. B 36145

[31] Veneziano G 1991 Phys. Lett. B 265207 